\section{Psychological reactions and quality of life in patients with posterior uveal melanoma treated with ruthenium plaque therapy or enucleation: a one year follow-up study}

YVONNE BRANDBERG, ERIK KOCK, KRISTI OSKAR, EVA AF TRAMPE, STEFAN SEREGARD

\begin{abstract}
Purpose To investigate psychological reactions and quality of life among patients with posterior uveal melanoma.

Methods Consecutive patients with uveal

quality of life and emotional problems, but more problems with visual impairment. These differences in impact on disease-related functioning should be taken into account when treatment options are discussed.
\end{abstract} malignant melanoma (99/106), referred to St Erik's Eye Hospital 1995-1996, treated with rutheniuim plaque radiotherapy $(n=50)$ or enucleation $(n=49)$, were included in this non-randomised prospective comparative study. Questionnaires were completed before treatment (Hospital Anxiety and Depression Scale, HAD scale) and 2 and 12 months after diagnosis including the HAD scale, the Impact of Event Scale and the EORTC QLQ-C30.

A disease-specific questionnaire was included 12 months after diagnosis. Between-group differences were analysed by chi-square, Student's $t$-test and ANOVA.

Results A majority of the patients reported reduced 'Quality of Life' (72-85\%), 'Emotional functioning' (60-74\%) and 'Cognitive functioning' (51-61\%). 'Fatigue' was the most frequently reported symptom (61-72\%) followed by 'Insomnia' (43-58\%). Anxiety and depressive symptoms were relatively frequent up to 1 year after treatment, but the levels of anxiety decreased during the first year after treatment. Disease and treatment-related problems were reported in both treatment groups 1 year after diagnosis. Enucleated patients had more problems with appearance and judging distances, whereas those treated with radiotherapy reported vision impairment to a higher extent.

Conclusions Enucleated patients reported high levels of emotional distress, problems with appearance and judging distances during the first year after treatment. Patients treated with radiotherapy reported similar levels of
Key words Anxiety, Depression, Enucleation, Psychology, Quality of life, Ruthenium plaque radiotherapy, Uveal melanoma

Malignant melanoma of the posterior uvea is the most common primary intraocular malignancy, with a 10 year melanoma-related mortality rate of nearly $50 \% .{ }^{1}$ Uveal melanoma may often be managed by any of a range of eyepreserving options and in Sweden ruthenium plaque radiotherapy is currently used for most small, medium-sized and some large tumours. ${ }^{2}$ Whereas cycling tumour cells may remain after plaque radiotherapy, ${ }^{3}$ non-randomised data suggest that survival following radiotherapy equals that after enucleation. ${ }^{2,4}$ Valuable visual function is preserved in many eyes managed with plaque radiotherapy, ${ }^{2}$ but apparently most patients retain satisfactory visual performance status for many years regardless of whether treatment was plaque radiotherapy or enucleation. ${ }^{5,6}$

Despite the growing interest in quality of life following treatment for a variety of cancer diagnoses, ${ }^{7}$ little is known about either the psychosocial impact of uveal melanoma or when and why psychosocial dysfunction may occur. ${ }^{8}$ Recently, there has been a growing interest in the literature for the quality of life of patients with uveal melanoma. ${ }^{9-11}$ The importance of assessing quality of life and the functional impact of uveal melanoma was stressed in a recent editorial. ${ }^{12}$ To our knowledge, no prospective study of quality of
Y. Brandberg

Department of Oncology

Karolinska Hospital

Stockholm, Sweden

E. Kock

K. Oskar

E. af Trampe

S. Seregard

Ophthalmic Pathology and

Oncology Service

St Erik's Eye Hospital

Stockholm, Sweden

Yvonne Brandberg, PhD Department of Oncology Karolinska Hospital

S-171 76 Stockholm, Sweden

Tel: +46 851772422

Fax: +46 8326113

e-mail:

yvonne.brandberg@ce.ks.se

The study was financed by the Cancer Society in

Stockholm, grant NR. 96149

Received: 1 February 2000 Accepted in revised form: 8 June 2000 
life and psychological reactions to uveal melanoma has been published. In a retrospective study of quality of life in patients with choroidal melanoma, patients treated with enucleation showed higher levels of emotional distress and lower levels of vitality than those treated with ruthenium plaque radiotherapy about 5 years after treatment. ${ }^{11}$ Improved knowledge of the psychological impact of diagnosis and treatment may assist in determining the preferred management and refining post-operative routines for patients with uveal melanoma. $^{12}$

The aim of the study was to prospectively investigate psychological reactions and the quality of life in patients with uveal melanoma at the time of diagnosis and 2 and 12 months after treatment.

\section{Materials and methods}

\section{Patients}

All patients referred for posterior uveal melanoma to the Ophthalmic Pathology and Oncology Service, St Erik's Eye Hospital, Stockholm and primarily managed 1995-1996 with enucleation (E) or ruthenium plaque radiotherapy $(R)$ were eligible for inclusion in the present study.

Following clinical examination and relevant diagnostic procedures, patients were informed of the clinical diagnosis and given the benefits and disadvantages associated with any potential treatment (usually plaque radiotherapy or enucleation) according to the procedures described by Damato. ${ }^{12}$ The individual patient was then recommended a particular treatment and, briefly, patients with tumours $<7 \mathrm{~mm}$ in height and $<18 \mathrm{~mm}$ in diameter were advised to have plaque radiotherapy, whereas larger tumours were managed by enucleation. However, the actual choice was made jointly by the patient and ophthalmologist based upon the information available and the patient's preferences.

In general, patients selected for enucleation had larger tumours (median diameter $14 \mathrm{~mm}$; range 6-25 $\mathrm{mm}$ ) than patients receiving plaque radiotherapy (median diameter $8 \mathrm{~mm}$; range $4-16 \mathrm{~mm}$ ). Similarly, patients with enucleation had thicker tumours (median thickness $9 \mathrm{~mm}$; range 2-20 $\mathrm{mm}$ ) than patients with tumours managed with brachytherapy (median thickness $4 \mathrm{~mm}$; range $2-8 \mathrm{~mm}$ ). While 5 patients with ciliary body melanomas decided to have the eye removed, only 1 patient with a ciliary body tumour had plaque radiotherapy.

Before treatment was initiated, a liver ultrasound scan was performed and liver enzymes checked for signs of metastatic disease. Enucleation was conducted at a hospital near the patient's home, and at the time of the study, follow-up was based on local routines. Patients enucleated at St Erik's Eye Hospital had at least one follow-up examination 1 month after enucleation, at which time additional follow-up was planned depending on the patient's choice. All the study patients receiving plaque radiotherapy were treated at St Erik's Eye Hospital. These patients had four follow-up examinations during the first post-operative year and were then followed annually. Periodic follow-up for signs of metastatic disease includes liver monitoring semi-annually for 5 years.

\section{Procedure}

The patients were informed verbally and in writing about the study. Informed consent was obtained verbally. The patients were asked to complete questionnaires on three occasions. At the first visit to St Erik's Eye Hospital, before diagnosis was confirmed and appropriate management was decided, the patients received a questionnaire concerning anxiety and depression. Two months (range 1-3 months) after treatment the patients were interviewed by 'phone by a psychologist and asked to complete mailed questionnaires measuring quality of life, anxiety, depressive symptoms and subjective stress responses. A similar set of questionnaires, including an additional questionnaire regarding eye-specific symptoms, was mailed to patients 1 year after surgery.

\section{The instruments}

The Hospital Anxiety and Depression (HAD) Scale was developed to measure anxiety and depressive symptoms among patients with somatic diseases. ${ }^{13}$ It consists of 14 items -7 for anxiety and 7 for depressive symptoms assessing problems during the previous week in one of four categories (scored 0 to 3 ). The sum of scores was calculated for each scale (maximum 21).

The reliability and validity of the HAD scale have been tested in a number of international studies. ${ }^{14}$ Two cut-off scores have been recommended: $7 / 8$ for possible and 10/11 for probable clinical levels of anxiety and depression. ${ }^{13}$ The HAD scale was translated into Swedish and validated by Marianne Sullivan. ${ }^{15}$

The European Organisation for Research and Treatment of Cancer (EORTC) QLQ-C30 (+3) questionnaire has been developed by the EORTC Quality of Life Group for the measurement of quality of life in cancer patients in clinical trials. ${ }^{16}$ It consists of 33 items, incorporating nine multi-item scales: five functional scales (physical, role, cognitive, emotional and social); three symptom scales (fatigue, pain and nausea/vomiting); and three global health and quality of life scales. For each item, the respondent is asked to indicate on a four-point scale from 1 (Not at all) to 4 (Very much) the extent to which he or she has experienced the problem during the past week. A number of single-item scales are also included.

The Impact of Event Scale (IES) was included in order to measure the post-traumatic stress responses to the diagnosis of ocular melanoma and the eye operation. The IES was developed by Horowitz and co-workers ${ }^{17}$ in order to measure the degree of subjective stress experienced as a result of a specific event. ${ }^{18}$ The IES consists of 15 items, and taps two dimensions paralleling the definition of the DSM-III 'Post Traumatic Stress Disorder'. ${ }^{19}$ 'Avoidance responses' (8 items) includes 
ideational restrictions, denial of the impact of the event, blunted sensation, behavioural inhibition including awareness of emotional numbness and counterphobic activities. 'Intrusion responses' (7 items) includes unbidden thoughts and images, dreams, strong waves of feelings and repetitive behaviour. The patients indicated the frequency of endorsement of a given experience the preceding week on a four-point scale. Cut-off scores of low $(0-8)$, medium (9-19) and high $(\geqslant 20)$ levels of distress have been suggested. ${ }^{20}$

The Eye Symptom Questionnaire. A literature review in 1994 revealed no existing standardised questionnaire including items assessing problems specific for patients with uveal melanoma. ${ }^{21} \mathrm{~A}$ list of items was constructed after interviewing the patients in this study 2 months after surgery and after reviewing the literature concerning quality of life and functional problems related to uveal melanoma and enucleation. Three physicians and one nurse, dedicated to the care of patients with uveal melanoma at St Erik's Eye Hospital in Stockholm, were then asked to read and comment on the list. The list was adapated according to their comments and 15 patients were then asked to complete the questionnaire and to comment on the content, phrasing of items, and whether any items should be deleted or added. This procedure resulted in a questionnaire including 15 items concerning: itching, irritation and pain in the operated eye (4 items), ability to read ( 2 items), vision impairment ( 2 items), problems with appearance ( 2 items), double vision ( 1 item), watching television (1 item), discharge from the eye (1 item), walking on uneven grounds ( 1 item) and problems driving a car (1 item). An item concerning problems with the prosthesis was added to the questionnaire for enucleated patients. The questionnaire had the same time frame and response format as the EORTC QLQ-C30. This work forms the basis of an 'ophthalmic module' currently developed by the EORTC Quality of Life Group.

The study was approved by the Karolinska Hospital Ethics Committee, nr. 94-332.

\section{Statistical methods}

Scoring of the questionnaires (HAD scale, IES and EORTC QLQ-C30 (+3)) was performed according to published guidelines. ${ }^{14,17,22}$

The standard deviations obtained for the EORTC QLQ-C30 (+3) and the IES varied within the treatment groups. Change in scores from one point of measurement to the next is considered to be less sensitive to variations in means. Therefore analyses of between-groupp (ruthenium plaque radiotherapy vs enucleation) differences over time were performed by Student's $t$-test for instruments used at two assessments.

To analyse differences between treatments, changes over time and interactions between treatment and point of assessment on the HAD scale, analysis of variance (ANOVA), repeated measurement was used. Differences between the treatments (ruthenium plaque radiotherapy vs enucleation) concerning disease-specific problems were analysed by chi-square test of differences in proportions of patients reporting problems.

The limit for statistically significant results was set to $p \leqslant 0.01$.

\section{Results}

A total of 106 patients with uveal malignant melanoma were eligible, referred to St Erik's Eye Hospital for a decision about treatment. Seven patients were not included ( 3 missed, 2 were psychiatrically insufficient, 1 refused, 1 lived abroad), leaving 99 included patients (93\%): 51 women (mean age 63, range $27-88$ years) and 48 men (mean age 64 , range $31-87$ years). The distribution of treatments across gender is shown in Table 1 . There were no statistically significant differences in sex or age between treatment groups. A total of 85 patients (86\%) (38 enucleated (E) and 47 treated with ruthenium plaque radiotherapy $(\mathrm{R})$ ) responded to the questionnaires about 2 months after treatment. The reasons for not responding were: metastatic disease (5 E), unwillingness to participate in the study (4 patients), change of treatment or not receiving treatment within 3 months from inclusion ( 3 patients) and old age (one woman born in 1907, one man born in 1910). At 12 months 78 patients responded: $32 \mathrm{E}$ and $46 \mathrm{R}$. Nine patients $(8 \mathrm{E}, 1 \mathrm{R})$ had died and 12 (9 E, $3 \mathrm{R})$ did not return the questionnaires. Reasons for not responding were unwillingness to participate (8 patients), old age (87 and 88 years old) (2 patients), change of treatment (1 patient) and administrative failure (1 patient). There were no statistical differences in mean age or in the distribution of gender between the first and third assessment.

\section{Quality of life}

The means and standard deviations (SD) on the EORTC QLQ-C30 functional and symptom scales, and for individual items, are presented in Table 2. There were no statistically significant differences between the groups with respect to quality of life (QoL). 'Role functioning' increased significantly during the first year after surgery in both groups $[F(67,1)=26.2, p<0.001](\mathrm{E} 19.6 ; \mathrm{R} 11.0)$. There were only minor changes $(<6.0)$ for the other subscales.

Table 1. Treatment and gender: number (\%) of patients

\begin{tabular}{lccc}
\hline & $\begin{array}{c}\text { Ruthenium } \\
n(\%)\end{array}$ & $\begin{array}{c}\text { Enucleation } \\
n(\%)\end{array}$ & $\begin{array}{c}\text { Total } \\
n(\%)\end{array}$ \\
\hline Women & $24(47)$ & $27(53)$ & $51(52)$ \\
Men & $26(54)$ & $22(46)$ & $48(48)$ \\
Total & $50(51)$ & $49(49)$ & 99 \\
\hline
\end{tabular}


Table 2. EORTC QLQ-C30 (+3) scale and single item scores: means and standard deviations (SD) at two points of assessment

\begin{tabular}{|c|c|c|c|c|c|c|c|c|}
\hline & \multicolumn{4}{|c|}{ Two months } & \multicolumn{4}{|c|}{ One year } \\
\hline & \multicolumn{2}{|c|}{$\begin{array}{c}\text { Enucleation } \\
\quad(n=38)\end{array}$} & \multicolumn{2}{|c|}{$\begin{array}{c}\text { Ruthenium } \\
(n=47)\end{array}$} & \multicolumn{2}{|c|}{$\begin{array}{c}\text { Enucleation } \\
\quad(n=32)\end{array}$} & \multicolumn{2}{|c|}{$\begin{array}{l}\text { Ruthenium } \\
(n=46)\end{array}$} \\
\hline & Mean & SD & Mean & SD & Mean & $\mathrm{SD}$ & Mean & $\mathrm{SD}$ \\
\hline \multicolumn{9}{|l|}{ Functional scales ${ }^{a}$} \\
\hline Physical (PF) & 87 & 18 & 87 & 20 & 88 & 21 & 85 & 22 \\
\hline Role (RFn) & 74 & 28 & 82 & 25 & 93 & 10 & 94 & 10 \\
\hline Emotional (EF) & 70 & 30 & 82 & 19 & 73 & 28 & 77 & 25 \\
\hline Cognitive $(\mathrm{CF})$ & 80 & 23 & 82 & 23 & 77 & 26 & 80 & 22 \\
\hline Social (SF) & 81 & 21 & 89 & 20 & 85 & 24 & 83 & 25 \\
\hline Overall QoL & 68 & 23 & 72 & 18 & 69 & 28 & 74 & 22 \\
\hline \multicolumn{9}{|l|}{ Symptom scales ${ }^{b}$} \\
\hline Fatigue (FA) & 21 & 25 & 21 & 23 & 24 & 27 & 24 & 22 \\
\hline Nausea/vomiting (NV) & 3 & 6 & 1 & 6 & 3 & 8 & 2 & 8 \\
\hline Pain (PA) & 16 & 26 & 9 & 18 & 13 & 21 & 13 & 21 \\
\hline \multicolumn{9}{|l|}{ Single items ${ }^{b}$} \\
\hline Dyspnoea (DY) & 11 & 19 & 16 & 26 & 17 & 21 & 19 & 26 \\
\hline Insomnia (SL) & 28 & 32 & 19 & 26 & 30 & 32 & 21 & 24 \\
\hline Appetite loss (AP) & 7 & 14 & 6 & 15 & 7 & 17 & 9 & 20 \\
\hline Constipation (CO) & 12 & 21 & 2 & 8 & 11 & 22 & 9 & 22 \\
\hline Diarrhoea (DI0 & 5 & 16 & 3 & 9 & 5 & 12 & 4 & 13 \\
\hline Economic impact (FI) & 15 & 29 & 12 & 27 & 12 & 5 & 13 & 22 \\
\hline
\end{tabular}

${ }^{a}$ Score from 0 to 100 , higher scores indicating higher levels of functioning.

${ }^{b}$ Scored from 0 to 100 , higher scores indicating higher levels of problems.

The patients were also divided in two groups for each functional and symptom scale and for the single items included in EORTC QLQ-C30 (+3) dependent on whether they reported reduced function and symptoms (Table 3).

The proportions of patients reporting reduced functioning and having symptoms remained during the first year after surgery with the exception of 'Role functioning', where the proportions reporting problems decreased in both groups. A majority of the patients experienced reduced QoL (72-85\%), 'Emotional functioning' (60-74\%) and 'Cognitive functioning' (51-61\%). 'Fatigue' was the most frequently reported symptom (61-72\%) followed by 'Insomnia; (43-58\%).

Least common was 'Nausea/vomiting' and 'Diarrhoea', reported by about $10 \%$.

Table 3. Number of patients (\%) reporting reduction in function and symptoms on EORTC QLQ-C30 subscales and single items

\begin{tabular}{|c|c|c|c|c|c|c|c|c|}
\hline & \multicolumn{4}{|c|}{ Two months } & \multicolumn{4}{|c|}{ One year } \\
\hline & \multicolumn{2}{|c|}{$\begin{array}{l}\text { Enucleation } \\
(n=37-38)\end{array}$} & \multicolumn{2}{|c|}{$\begin{array}{l}\text { Ruthenium } \\
(n=46-47)\end{array}$} & \multicolumn{2}{|c|}{$\begin{array}{l}\text { Enucleation } \\
(n=29-31)\end{array}$} & \multicolumn{2}{|c|}{$\begin{array}{l}\text { Ruthenium } \\
(n=42-43)\end{array}$} \\
\hline & $n$ & $\%$ & $n$ & $\%$ & $n$ & $\%$ & $n$ & $\%$ \\
\hline \multicolumn{9}{|l|}{ Functional scales ${ }^{a}$} \\
\hline Physical (PF) & 14 & 37 & 16 & 35 & 11 & 36 & 16 & 38 \\
\hline Role (RFn) & 22 & 58 & 22 & 48 & 9 & 29 & 12 & 28 \\
\hline Emotional (EF) & 28 & 74 & 31 & 66 & 21 & 68 & 26 & 60 \\
\hline Cognitive (CF) & 23 & 61 & 24 & 51 & 18 & 58 & 26 & 60 \\
\hline Social (SF) & 20 & 53 & 15 & 33 & 12 & 39 & 21 & 50 \\
\hline Overall QoL & 30 & 79 & 40 & 85 & 21 & 72 & 32 & 74 \\
\hline \multicolumn{9}{|l|}{ Symptom scales ${ }^{b}$} \\
\hline Fatigue (FA) & 25 & 66 & 28 & 61 & 21 & 68 & 31 & 72 \\
\hline Nausea/vomiting (NV) & 6 & 16 & 3 & 6 & 4 & 13 & 3 & 7 \\
\hline Pain (PA) & 15 & 39 & 11 & 23 & 11 & 35 & 15 & 35 \\
\hline \multicolumn{9}{|l|}{ Single items ${ }^{b}$} \\
\hline Dyspnoea (DY) & 11 & 29 & 17 & 36 & 13 & 30 & 18 & 42 \\
\hline Insomnia (SL) & 22 & 58 & 20 & 43 & 17 & 55 & 22 & 51 \\
\hline Appetite loss (AP) & 8 & 21 & 8 & 17 & 6 & 19 & 9 & 21 \\
\hline Constipation (CO) & 11 & 29 & 3 & 6 & 8 & 26 & 7 & 16 \\
\hline Diarrhoea (DI0 & 4 & 11 & 4 & 9 & 4 & 13 & 4 & 9 \\
\hline Economic impact (FI) & 10 & 26 & 10 & 21 & 8 & 26 & 13 & 31 \\
\hline
\end{tabular}

${ }^{a}$ Scored from 0 to 100 , higher scores indicating higher levels of functioning.

${ }^{b}$ Scored from 0 to 100 , higher scores indicating higher levels of problems. 
Table 4. HAD anxiety and depression subscales: means and standard deviations (SD) at three points of assessment

\begin{tabular}{|c|c|c|c|c|c|c|c|c|c|c|c|c|}
\hline & \multicolumn{4}{|c|}{ Pre-treatment } & \multicolumn{4}{|c|}{ Two months after treatment } & \multicolumn{4}{|c|}{ One year after treatment } \\
\hline & \multicolumn{2}{|c|}{ Enucleation } & \multicolumn{2}{|c|}{ Ruthenium } & \multicolumn{2}{|c|}{ Enucleation } & \multicolumn{2}{|c|}{ Ruthenium } & \multicolumn{2}{|c|}{ Enucleation } & \multicolumn{2}{|c|}{ Ruthenium } \\
\hline & Mean & SD & Mean & $\mathrm{SD}$ & Mean & SD & Mean & SD & Mean & $\mathrm{SD}$ & Mean & SD \\
\hline Anxiety $^{a}$ & 8.7 & 5.1 & 6.6 & 4.9 & 5.6 & 4.1 & 4.5 & 4.3 & 5.8 & 4.7 & 5.0 & 4.7 \\
\hline Depression $^{a}$ & 4.3 & 3.4 & 3.6 & 2.6 & 4.3 & 3.8 & 3.7 & 3.5 & 4.5 & 4.2 & 4.0 & 3.9 \\
\hline
\end{tabular}

${ }^{a}$ Scored from 0 to 21 , higher scores indicating higher levels of problems.

\section{Anxiety and depressive symptoms}

Mean scores on the HAD anxiety and depression subscales before treatment and 2 months and 1 year after treatment, are presented in Table 4.

HAD anxiety decreased between the points of assessment $[F(2,64)=16.0 p<0.001]$, but not HAD depressive symptoms $[F(1,81)=6.55, p<0.05]$. No between-group differences were found.

The patients were categorised as having clinical levels of anxiety or depression according to their scores on the HAD subscales in groups 'No case' (0-7), 'Possible clinical case' (8-10) and 'Clinical case' (> 10) (Table 5). There were no statistically significant differences between the treatment groups in the proportion of patients in each category (chi-square).

\section{Subjective stress responses}

The IES scale measured the degree of subjective stress, experienced as a result of the cancer diagnosis and eye operation, as well as response styles. The means and SD for the subscales at two points of assessment are presented in Table 6. No differences in change scores were found between the treatment groups.

The numbers and proportions of patients scoring in the categories 'Low' (0-8), 'Medium' (9-19) and 'High' $(\leqslant 20)$ on the IES subscales are shown in Table 7 . No statistically significant differences in proportions scoring in the three categories for each of the subscales were found.

\section{Problems related to uveal melanoma and its treatment}

At the 1 year follow-up assessment, the patients responded to a questionnaire about disease-specific problem areas. The proportions of patients in each treatment group reporting problems are presented in Fig 1 . The most commonly reported problems were 'Vision impairment' (difficulty seeing at the side, 73\%), 'Walking on uneven ground' (67\%), 'Pain in the eye' (63\%) and 'Discharge from the eye' (53\%). Statistically significant higher proportions of enucleated patients had problems walking on uneven ground (chi-square $=10.9$, d.f. $=1, p<0.01$ ) and with their appearance (chi-square $=$ 10.5 , d.f. $=1, p<0.01$ ) as compared with the radiotherapy group. The opposite was found for vision impairment, where a higher proportion among those receiving radiotherapy reported problems (chi-square $=6.5$, d.f. $=1, p<0.01)$.

\section{Discussion}

In the present study, quality of life, emotional distress and disease-related effects after treatment of uveal melanoma were studied prospectively in a sample of consecutive patients. Compared with patients with other cancer diagnoses, and with normative data, high proportions of patients reported reduced quality of life and substantial emotional problems 1 year after the surgery. ${ }^{23-28}$ Only abilities to perform daily activities, such as work and hobbies, increased. Thus the majority of the problems reported by patients after surgery remain, at a time when the patients are expected to have returned to their ordinary capacity.

Emotional problems were more pronounced among the enucleated patients, although not statistically significant, probably due to small sample size. Our result is consistent with the finding in a retrospective study from the United States showing higher levels of emotional problems persisting up to 5 years after treatment among enucleated patients as compared with patients treated with ruthenium plaque therapy. ${ }^{11}$

Table 5. Number (\%) of patients scoring in categories 'No case' (<7), 'Possible clinical case' (8-10) and 'Clinical case' (>10) on the Hospital Anxiety and Depression subscales

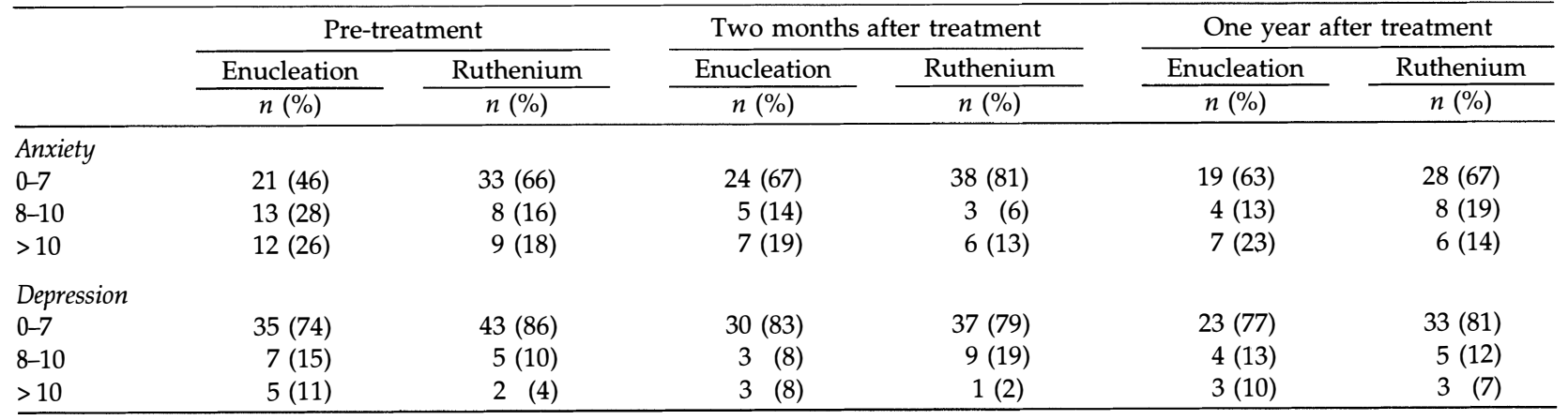


Table 6. IES intrusion and avoidance subscales: means and standard deviations (SD) at two points of assessment

\begin{tabular}{|c|c|c|c|c|}
\hline & \multicolumn{2}{|c|}{ Two months after treatment } & \multicolumn{2}{|c|}{ One year after treatment } \\
\hline & Enucleation & Ruthenium & Enucleation & Ruthenium \\
\hline & Mean (SD) & Mean (SD) & Mean (SD) & Mean (SD) \\
\hline Intrusion $^{a}$ & $13.2(7.7)$ & $9.6(9.1)$ & $11.6 \quad(9.9)$ & $9.7(8.6)$ \\
\hline Avoidance $^{b}$ & $15.6(10.4)$ & $13.0(10.7)$ & $14.1(10.2)$ & $11.7(8.6)$ \\
\hline
\end{tabular}

${ }^{a}$ Scored from 0 to 35 , higher scores indicating higher levels of intrusion.

${ }^{b}$ Scored from 0 to 40 , higher scores indicating higher levels of avoidance.

Nearly half the patients reported high levels of anxiety at the first visit to St Erik's Eye Hospital. This finding probably reflects the uncertainty the patients experienced before diagnosis was confirmed and their treatment was decided. Considering this high level of emotional distress, information to the patients should be carefully planned in order for the patients to receive the message. The high levels of emotional distress also limit the patients' abilities to make informed decisions at this point. Preferably, patients should have the opportunity to carefully consider the treatment alternatives and the consequences of the treatment alternatives. ${ }^{12}$

There might be several explanations for the high levels of emotional distress among patients with uveal melanoma as compared with those with cutaneous melanoma. Firstly, the majority of the patients with cutaneous melanoma had no remaining problems after surgery, as opposed to the uveal melanoma patients, who reported impairments with respect to visual functioning. In addition, every time these patients look in the mirror, they will be reminded of their cancer disease and many of them reported problems with appearance, also reflected by the high level of intrusive thoughts. Secondly, the follow-up routines differ. Patients with cutaneous melanoma are followed regularly at the Department of Oncology, with intervals dependent on tumour thickness. Patients treated with ruthenium plaque therapy are followed every third month at St Erik's Eye Hospital, and they reported slightly fewer problems than those who had been enucleated. Followup routines for the enucleated patients differed, and were performed according to local policies. Some of these patients only had one follow-up after surgery and were then left with their concerns. They were instructed, should problems arise, to contact the clinic where the enucleation had been performed. As these patients have the largest tumours and the greatest risk of recurrence, routine follow-ups with thorough physical examinations might reveal some of their problems and lessen their responsibility to detect metastatic disease themselves. The follow-ups also provide an opportunity to ask questions and to discuss functional and emotional matters, and problems with the prosthesis. A third explanation is that patients with cutaneous melanoma stage 1 are informed of their favourable prognosis, whereas the prognosis is worse for patients with uveal melanoma, and especially for those with large tumours.

The patients in both treatment groups reported disease- and treatment-related problems 1 year after treatment, although the 'profile' of problems differed. Problems with appearance and judging distances were more common among enucleated patients, whereas problems with vision impairment were reported more frequently by those who received radiotherapy. Patients should be informed of the different impact of the two treatments, in order to make more informed decisions when there is an option to choose between them.

Ideally, effects of treatments should be studied in randomised trials. It was not possible to randomise patients in the present study due to ethical reasons. Instead, the treatment chosen for each patient depended on medical variables and patient preferences. Those who preferred enucleation to ruthenium plaque radiotherapy were expected to be more positive to this kind of treatment compared with a hypothesised randomised sample of enucleated patients. On the other hand, enucleation was decided for some of the patients dependent on tumour location and size. Thus, the results of our study reflect patients undergoing standard procedures in deciding about treatment of uveal melanoma in Sweden.

Table 7. Number of patients (\%) scoring in three categories on the IES subscales

\begin{tabular}{|c|c|c|c|c|}
\hline & \multicolumn{2}{|c|}{ Two months after treatment } & \multicolumn{2}{|c|}{ One year after treatment } \\
\hline & Enucleation & Ruthenium & Enucleation & Ruthenium \\
\hline & Mean (SD) & Mean (SD) & Mean (SD) & Mean (SD) \\
\hline \multicolumn{5}{|c|}{ Intrusion } \\
\hline $0-8$ & $10(28)$ & $26(57)$ & $14(50)$ & $22(50)$ \\
\hline 9-19 & $17(47)$ & $11(24)$ & $7(25)$ & $15(35)$ \\
\hline$\geqslant 20$ & $9(25)$ & $9(19)$ & $7(25)$ & $7(15)$ \\
\hline \multicolumn{5}{|c|}{ Avoidance } \\
\hline $0-8$ & $11(32)$ & $19(42)$ & $11(37)$ & 17 (39) \\
\hline 9-19 & $13(38)$ & $13(29)$ & $10(33)$ & $17(39)$ \\
\hline$\geqslant 20$ & $10(29)$ & $13(29)$ & $9(30)$ & $10(22)$ \\
\hline
\end{tabular}




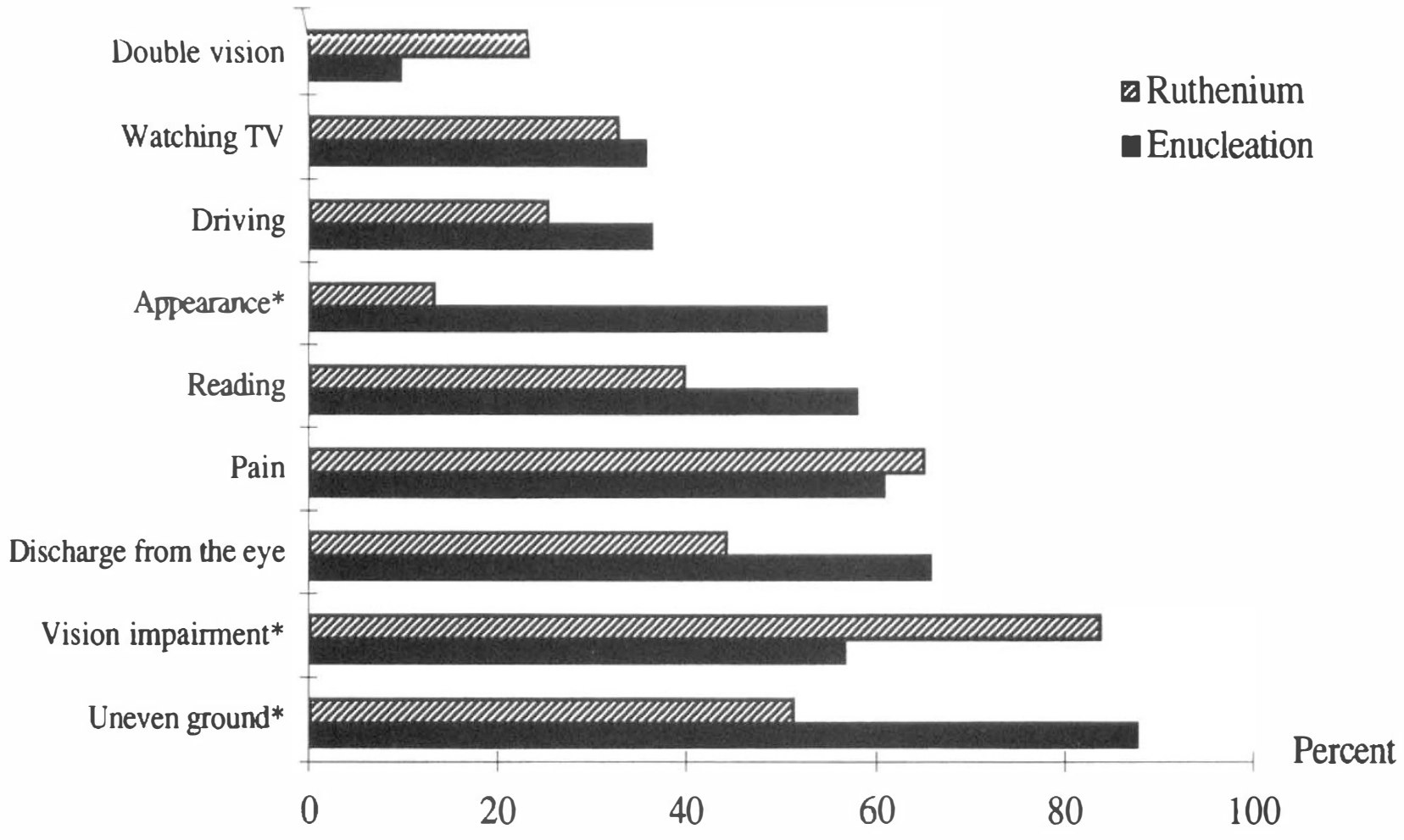

Fig. 1. Percentage of patients by treatment group reporting problems 1 year after treatment in areas related to uveal melanoma. *Significant difference $(\mathrm{p}<0.01)$ between the ruthenium plaque and enucleation groups.

Although the number of patients in each treatment group is small, and despite the fact that this is not a randomised study, our results indicate persistent emotional distress among patients with uveal melanoma, especially among those treated with enucleation. The two treatments have a different impact on disease-related functioning and in cases where discussions about treatment options are appropriate, our results can be of use in helping the doctor and the patient to make informed decisions about which treatment to choose.

\section{References}

1. Seregard S. Posterior uveal melanoma: the Swedish perspective. Acta Ophthalmol Scand 1996;74:315-29.

2. Seregard S, af Trampe E, Lax I, et al. Results following episcleral plaque radiotherapy for posterior uveal melanoma. Acta Ophthalmol Scand 1997;75:11-6.

3. Seregard S, Lundell G, Lax I, et al. Tumour cell proliferation after failed ruthenium plaque therapy for posterior uveal melanoma. Acta Ophthalmol Scand 1997;75:148-54.

4. Seregard S, Kock E. Prognostic indicators following enucleation for posterior uveal melanoma. Acta Ophthalmol Scand 1995;73:340-4.

5. Edwards MG, Schachat AP. Impact of enucleation for choroidal melanoma on the performance of visiondependent activities. Arch Ophthalmol 1991;109:519-21.

6. Augsberger JJ, Goel SD. Visual function following enucleation or episcleral plaque radiotherapy for posterior uveal melanoma. Arch Ophthalmol 1994;112:786-9.

7. Olschewski M, Schulgen G, Schumacher M, Altman DG. Quality of life assessment in clinical cancer research. Br J Cancer 1994;70:1-5.
8. Bunston T, Mackie A, Jones D, Mings D. Identifying the nonmedical concerns of patients with ocular melanoma. J Ophthalmic Nurs Technol 1994;13:227-37.

9. Albert DN. The ocular melanoma story. LII Edward Jackson Memorial Lecture: part II. Am J Ophthalmol 1997;123:729-41.

10. Hart PM, Chakravarthy U, Stevenson MR. Questionnairebased survey on the importance of quality of life measures in ophthalmic practice. Eye 1998;12:124-6.

11. Cruickshanks KJ, Fryback DG, Nondal DM, et al. Treatment choice and quality of life in patients with choroidal melanoma. Arch Ophthalmol 1999;117:461-7.

12. Damato B. Disease and 'dis-ease' in patients with uveal melanoma [editorial]. Br J Ophthalmol 2000;84:343-5.

13. Zigmond AS, Snaith RP. The Hospital Anxiety and Depression Scale. Acta Psychiatr Scand 1983;67:361-70.

14. Herrman C. International experiences with the Hospital Anxiety and Depression Scale: a review of validation data and clinical results. J Psychosom Res 1997;42:17-41.

15. Sullivan M, Karlsson J, Sjöström L, et al. Swedish obese subject (SOS): an intervention study of obesity. Baseline evaluation of health and psychosocial functioning in the first 1743 subjects examined. Int J Obes 1993;17:503-12.

16. Aaronson NK, Ahmedzai S, Bergman B, et al. The European organization for research and treatment of cancer QLQ-C30: a quality-of-life instrument for use in international clinical trials in oncology. J Natl Cancer Inst 1993;85:365-76.

17. Horowitz M, Wilner N, Alvarez W. Impact of Event Scale: a measure of subjective stress. Psychosom Med 1979;41:209-18.

18. Zielberg NJ, Weiss DS, Horowitz MJ. Impact of Event Scale: a cross-validation study and some empirical evidence supporting a conceptual model of stress response syndromes. J Consult Clin Psychol 1982;50:407-14.

19. American Psychiatric Association. Diagnostic and statistical manual of retinal disorders. 3rd edn. Washington, DC: APA, 1980. 
20. Horowitz M. Stress response syndromes and their treatment. In: Goldberger L, Breznitz S, editor. Handbook of stress: theoretical and clinical aspects. New York: The Free Press, 1982:711-32.

21. Foss AJE, Lamping DL, Schroter S, Hungerford J. Development and validation of a patient based measure of outcome in ocular melanoma. Br J Ophthalmol 2000;84:347-51.

22. EORTC Quality of Life Study Group. EORTC QLQ C-30 Scoring Manual. Brussels: EORTC Data Center, 1995.

23. Brandberg Y, Bolund C, Sigurdardottir V, et al. Anxiety and depression at different stages of malignant melanoma. Psycho-Oncology 1992;1:72-8.

24. Brandberg Y, Månsson-Brahme E, Ringborg U, Sjödén PO. Psychological reactions in patients with malignant melanoma. Eur J Cancer 1995;31:157-62.
25. Lisspers J, Nygren A, Söderman E. Hospital Anxiety and Depression Scale (HAD): some psychometric data for a Swedish sample. Acta Psychiatr Scand 1997;96:281-6.

26. Hjermstad MJ, Fayers PM, Bjørdal K, Kaasa S. Health related quality of life in the general Norwegian population assessed by the European Organization for Research and Treatment of Cancer Core Quality of Life Questionnaire: the QLQ-C30 (+3). J Clin Oncol 1998;16:1188-96.

27. Wisløff F, Hjort M, Kaasa S, Westin J. Effect of interferon on the health-related quality of life of multiple myeloma patients: results of a Nordic randomized trial comparing melphalan-prednisone to melphalan-prednisone $+\alpha$ interferon. Br J Haematol 1996;94:324-32.

28. Kaasa S, Malt U, Hagen S, et al. Psychological distress in cancer patients with advanced disease. Radiother Oncol 1993;27:126-34. 